\title{
The impact of parallel programming on faster image filtering
}

\author{
Kamil Książek $^{1}$, Zbigniew Marszałek ${ }^{1,2}$, Giacomo Capizzi ${ }^{2,1}$, \\ Christian Napoli ${ }^{3}$, Dawid Połap ${ }^{1}$, Marcin Woźniak ${ }^{1,2}$ \\ ${ }^{1}$ Institute of Mathematics, Silesian University of Technology, \\ Kaszubska 23, 44-100 Gliwice, Poland \\ 2 Department Electrical, Electronics and Informatics Engineering, \\ University of Catania, V. Andrea Doria 6, 95125 Catania, Italy \\ 3 Department of Mathematics and Computer Science, University of Catania, \\ V. Andrea Doria 6, 95125 Catania, Italy \\ Email: KamilKsiazek95@gmail.com,Zbigniew.Marszalek@polsl.pl, Gcapizzi@diees.unict.it, \\ Napoli@dmi.unict.it, Dawid.Polap@polsl.pl, Marcin.Wozniak@polsl.pl
}

\begin{abstract}
Parallel programming is a field of science with a great potential nowadays due to the development of advanced computers architectures. Appropriate usage of this tool can be therefore highly beneficial in multimedia applications and significantly decreases the time of calculations.

In this article, we analyze how the speed of calculations is influenced by the usage of parallel algorithms in image filtering processes. We present a method based on multithreading and the division of the image for rectangles. The filter is applied parallel on each part of the image. Results show that in some cases our proposition can bring over $90 \%$ benefit when compared to the classical approach.
\end{abstract}

Index Terms - parallel programming, image filtering, Laplacian, multithreading

\section{INTRODUCTION}

$\mathbf{P}$ ARALLEL programming is currently a dynamically growing field of computer science. Modern multicore processors enable a significant reduction of computation time. Proper use of computing power is an enormous challenge for programmers. A skillful preparation of parallel instructions that are to be carried out causes a lot of problems. However, benefits of parallel programming are obvious. Some multimedia applications require a huge amount of time - it is visible for instance in image processing. Filtering the images containing the several thousand pixels can take a lot of time, especially, when there is a substantial number of images to be filtered. Therefore an intelligent methods that improve image processing are very important.

It is clear that image filtering has a lot of applications. It is possible to improve the quality of photos with blur, noise or other undesirable effects. Moreover, sharpening the edges can be helpful in the objects detection. Parallel methods can be very useful in graphics processing and cloud computing directed to multithreading. High-quality images are also crucial in medicine in diagnosis of diseases (for instance in X-ray pictures). Therefore, capturing the details is very important. In this article we present multithreading in image filtering, and its impact on the whole process. Our approach is designed to equally distribute work among all the threads. The input image is divided into equal rectangles and each thread filters only the designated area. Therefore by the proposed algorithm we construct a method which uses all available cores. Depending on the number of CPU threads in the computer we can significantly decrease time of processing, reaching even $90 \%$ of improvement. It has a significant importance for HD multimedia systems where all the images and multimedia streams are very complex structures. Therefore our proposed method may reduce time and improve the efficiency of processing. For the research we have used an architecture with $32 \mathrm{CPU}$ threads and 320 GB of memory.

The main part of this article is following: Section II describes some related works, Section III presents a theoretical background of the image filtering and three Laplacian filters applicated in the research. In Section IV it is shown a detailed description of the tested parallel method. Section $\mathrm{V}$ gives a results of measurements and Section VI contains conclusions and remarks after studies.

\section{RELATED WORKS}

Very important application of multitasking is connected with data processing. In [1] it was shown how to parallelize fast sorting algorithm, while in [2] it was proposed a new more efficient parallel merge sort algorithm. These decrease computation time in large databases for instance in case of data analysis. In [3] an overview on intelligent systems for data retrieval was discussed. Graphics processing is frequent and very important topic of many publications. In [4] it was proposed a method how to more efficiently analyze the information from images for detection, and in [5] a segmentation of images based on graph analysis of the semantic image structure was presented. The image decomposition method which combines information from the infrared and visible images is presented in [6]. This method can be helpful for instance in target recognition. In [7] and [8] was presented a system for image data classification by the use of fast selection methods based on shapes comparisons. Authors in [9] propose a Weighted Guided Image Filtering algorithm (WGIF) which prevents so 
called "halo artefacts" effect. In many cases combination of different data ensures more efficient analysis. Such approach in medical images is shown in [10]. The method intended for filter identification is presented in [11]. An interesting problem connected with underwater imaging is introduced in [12]. Authors in [13] show the Core algorithm designed for document's identification from images. It is compared with classical detectors like ORB, SIFT and SURF-BRISK. In our paper it will be shown a method which can speed up the calculations on images. A properly and quickly processed image streamlines further work. The presented method is intended for initial processing of the images.

\section{IMAGE FILTERING}

A very common situation is that the analysis of the original image is difficult due to noise, blur or other factors. Furthermore, if the number of details is too large, a detection of the crucial parts of the image (for instance, the contours of presented objects) is impossible. Therefore it is necessary to pre-process the image. Further analysis will be easier thanks to such tools as the image filtering.

\section{A. Theoretical background}

One of the most popular color models is RGB model [14]. Each pixel consists of three components: $\mathrm{R}$ (red), $\mathrm{G}$ (green) and $\mathrm{B}$ (blue). They can be integers from the range $\{0,1, \ldots, 255\}$. For instance $[0,0,0]$ represents black, $[255,255,255]$ determines white, $[255,255,0]$ yellow, etc.

In this paper we assume that calculations will be performed by using the RGB model. Let $R_{n \times m}$ be a two-dimensional array with the values of pixels of a given image $(n$ is the width of an image and $m$ is the height of an image, $R[i, j]$ represents the position $(i, j)$ on the image, $i \in\{1, \ldots, n\}, j \in$ $\{1, \ldots, m\})$. The idea relies on modification of the image by moving the convolution mask over the pixels. The new values of three components $\mathrm{R}, \mathrm{G}, \mathrm{B}$ depend on the pixels in the nearest neighborhood of the calculated one. This process is illustrated in Fig. 1, by the example of $3 \times 3$ mask. The new value of the position $(x, y)$ depends on 9 pixels. Of course, other sizes are also allowed.

The greater the mask, the larger number of pixels is taken into account during processing. The impact of each pixel is defined by the table of weights, called a filter. In our research, there were applied three types of convolution masks: $3 \times 3$, $5 \times 5$ and $9 \times 9$. The larger the filter is, the more details are lost [15]. During calculations on larger masks it is necessary to create an auxiliary image with borders filled with black pixels (the convolution masks exceeds the original one). This operation has an nonsignificant influence on the final image but it enables the filtering. The pattern for the new value of each component of the pixel located at position $(i, j)$ in the case of $5 \times 5$ mask is as follows: [16]:

$$
R^{\prime}(i, j)=\frac{1}{M} \sum_{k=-2}^{2} \sum_{l=-2}^{2} w(k, l) \cdot R(i+k, j+l)
$$

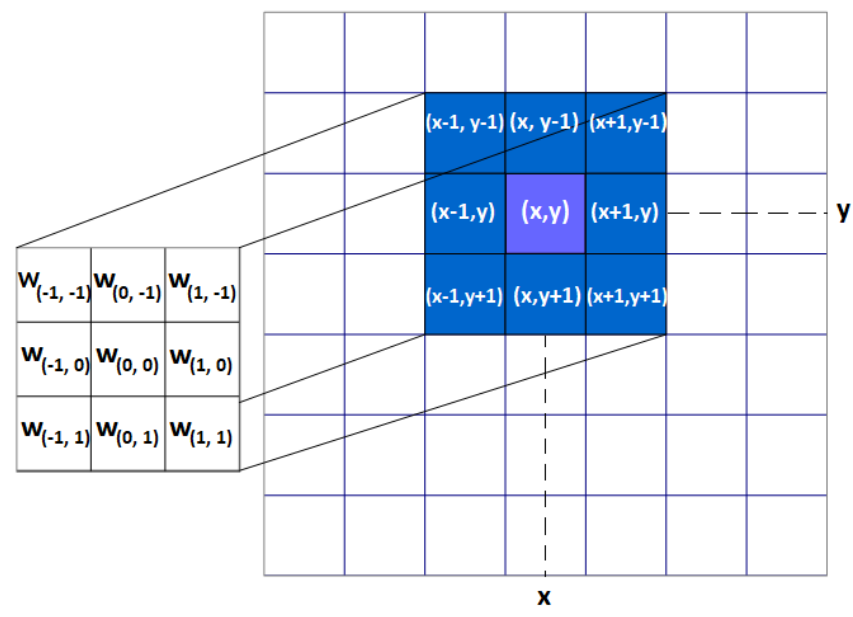

Fig. 1: The sample of using a $3 \times 3$ convolutional mask.

where $M$ is the sum of all values in the array (the convolution mask), $w(k, l)$ is the weight of pixel located at position $(i+k, j+l)$ and $R(i+k, j+l)$ is the previous value of the pixel at given position. Sometimes $M$ may be equal to 0 . In that situation, the factor $\frac{1}{M}$ is omitted. Patterns for other convolution masks are created similarly.

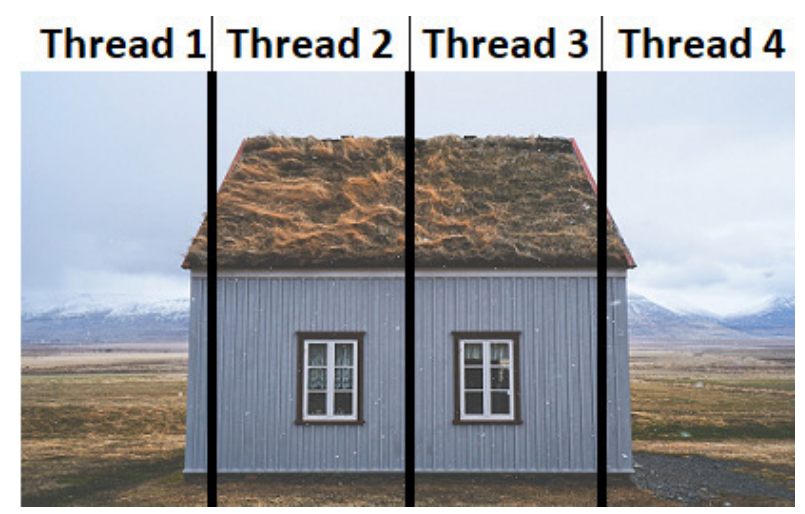

Fig. 2: Illustration of the image split into 4 rectangles. (The original photo was taken by Jonathan Andreo, and is available at unsplash.com)

\section{B. The applied filters}

During further calculations, three Laplacian filters will be used (Fig. 3). [17]. Their main task is sharpening the edges of the objects and hence, loosing of irrelevant details. The picture which has been filtered, is presented in Fig. 4. It is possible to see how the Laplacian filters influence the original image. The edges are therefore definitely more visible than the rest of the image. This kind of filters facilitates the detection of shapes.

\section{PARAllelization}

Image filtering involves a lot of calculations. The larger the photo is, the greater the time of filtering is. In case of analyzing a large number of pictures, minimizing the computation time is 


$$
\left(\begin{array}{ccc}
0 & -1 & 0 \\
-1 & 4 & -1 \\
0 & -1 & 0
\end{array}\right) \quad\left(\begin{array}{ccccc}
-1 & -1 & -1 & -1 & -1 \\
-1 & -1 & -1 & -1 & -1 \\
-1 & -1 & 24 & -1 & -1 \\
-1 & -1 & -1 & -1 & -1 \\
-1 & -1 & -1 & -1 & -1
\end{array}\right)
$$

(b) $5 \times 5$ Laplacian filter

$\left(\begin{array}{ccccccccc}0 & 1 & 1 & 2 & 2 & 2 & 1 & 1 & 0 \\ 1 & 2 & 4 & 5 & 5 & 5 & 4 & 2 & 1 \\ 1 & 4 & 5 & 3 & 0 & 3 & 5 & 4 & 1 \\ 2 & 5 & 3 & -12 & -24 & -12 & 3 & 5 & 2 \\ 2 & 5 & 0 & -24 & -40 & -24 & 0 & 5 & 2 \\ 2 & 5 & 3 & -12 & -24 & -12 & 3 & 5 & 2 \\ 1 & 4 & 5 & 3 & 0 & 3 & 5 & 4 & 1 \\ 1 & 2 & 4 & 5 & 5 & 5 & 4 & 2 & 1 \\ 0 & 1 & 1 & 2 & 2 & 2 & 1 & 1 & 0\end{array}\right)$

(c) $9 \times 9$ Laplacian filter

Fig. 3: The presentation of Laplacian filters applied in the paper.
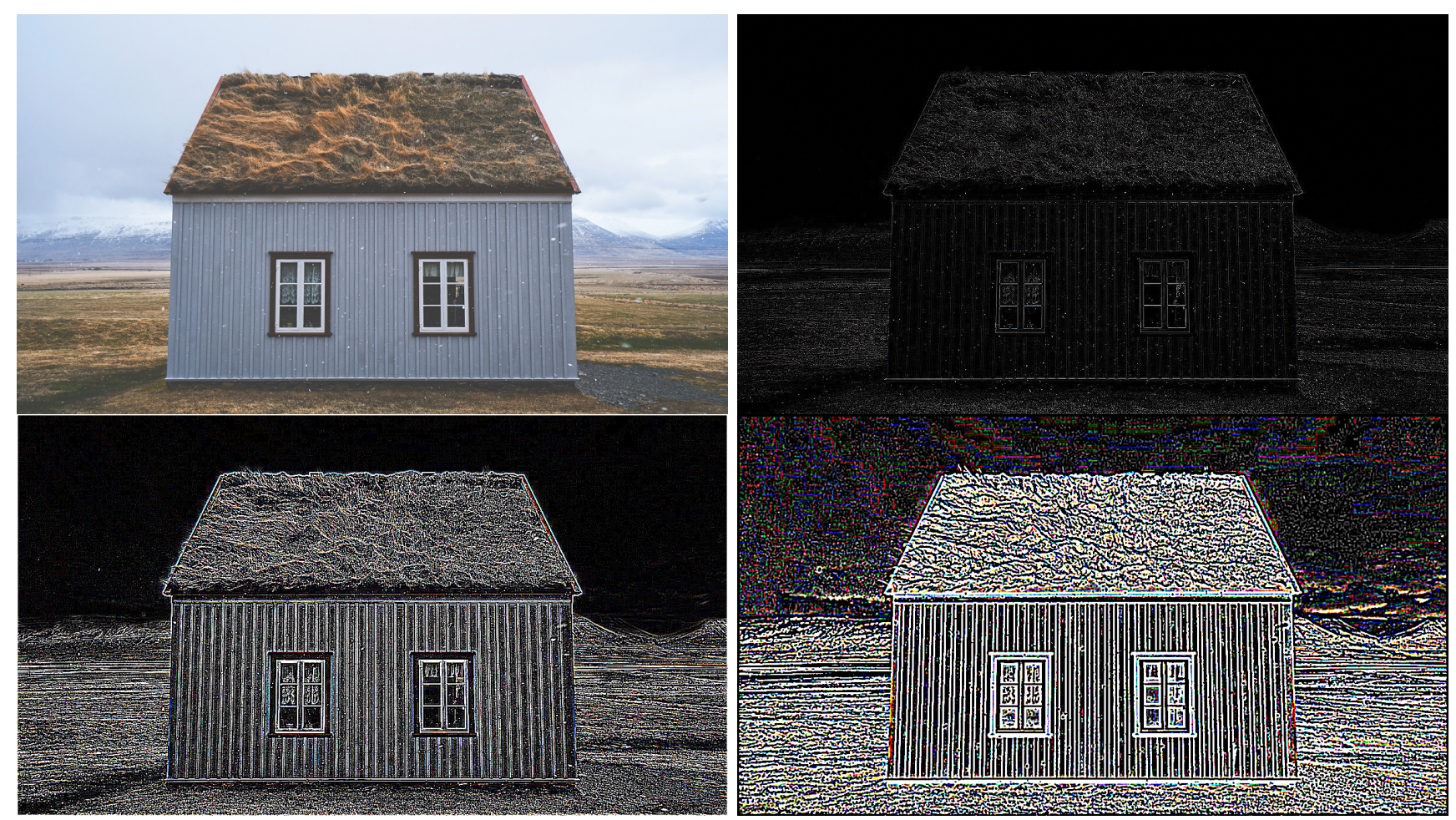

Fig. 4: The figure presents as follows: the original image and the image after $3 \times 3$ Laplacian filter (the first row) and images after $5 \times 5$ and $9 \times 9$ Laplacian filter (the second row). (The original photo was taken by Jonathan Andreo, and is available at unsplash.com)

crucial. The idea presented in this paper relies on moving the mask parallely over the image. The photo can be divided into $t$ rectangles, where $t$ is the number of threads (an exemplary division into 4 rectangles is presented in Fig. 2). Then each thread applies the filter to the allocated area and does not influence any other parts. Finally, all filtered fragments are combined into one image.

The only issue is to determine the way of assigning suitable areas for $t$ threads. Each thread after running receives its own index (the numbering starts from zero). Let $w$ be the width of the processed image (in pixels). Then the number of pixels per one thread is equal to $\left\lfloor\frac{w}{t}\right\rfloor$. The ceiling function is necessary because $\frac{w}{t}$ may not be an integer. In such a situation the last thread can have slightly more pixels to calculate. The
Algorithm 1 presents the pseudocode of the proposed method.

\section{EXPERIMENTAL RESULTS}

As was said before, application of three Laplacian filters was tested. In all cases, 100 measurements were performed and the results were averaged. The investigated image is 1200 pixels wide and 676 pixels high. For each convolution mask the image was filtered by using 1, 2, 4, 8, 16 and 32 threads. The algorithm was implemented in $\mathrm{C \#}$ language. The testing parallel architecture was Quad-Core AMD Opteron 8356 8p (32 CPU threads). Detailed results of experiments are presented in Tab. I and shown in Fig. 5 - 7. On all graphs the horizontal axis represents the number of threads and the vertical axis represents time. 
TABLE I: Results of image filtering by using Laplacian filters (100 averaged measurements).

\begin{tabular}{|c|c|c|c|c|c|}
\hline \multicolumn{6}{|c|}{$3 \times 3$ convolution mask } \\
\hline threads & $\begin{array}{ll}\text { average } & \text { time } \\
\text { (seconds) }\end{array}$ & percentage & $\begin{array}{l}\text { standard } \\
\text { deviation }\end{array}$ & $\begin{array}{l}\text { average time } \\
\text { (CPU ticks) }\end{array}$ & $\begin{array}{l}\text { standard } \\
\text { deviation }\end{array}$ \\
\hline 1 & 17.87600 & $100 \%$ & 0.23354 & 40153258 & 524569 \\
\hline 2 & 10.18082 & $56.95 \%$ & 0.30065 & 22868252 & 675319 \\
\hline 4 & 6.03999 & $33.79 \%$ & 0.28318 & 13567088 & 636091 \\
\hline 8 & 3.84961 & $21.54 \%$ & 0.25843 & 8647022 & 580477 \\
\hline 16 & 2.93947 & $16.44 \%$ & 0.21808 & 6602667 & 489848 \\
\hline 32 & 2.42189 & $13.55 \%$ & 0.08085 & 5440081 & 181596 \\
\hline \multicolumn{6}{|c|}{$5 \times 5$ convolution mask } \\
\hline 1 & 46.05641 & $100 \%$ & 1.08065 & 103452367 & 2427362 \\
\hline 2 & 24.74928 & $53.74 \%$ & 0.26110 & 55592085 & 586472 \\
\hline 4 & 14.43128 & $31.33 \%$ & 0.26109 & 32415682 & 1912613 \\
\hline 8 & 8.82084 & $19.15 \%$ & 0.97053 & 19813459 & 2180018 \\
\hline 16 & 6.05791 & $13.15 \%$ & 0.49399 & 13607337 & 1109609 \\
\hline 32 & 4.32978 & $9.40 \%$ & 0.15660 & 9725596 & 351746 \\
\hline \multicolumn{6}{|c|}{$9 \times 9$ convolution mask } \\
\hline 1 & 141.72927 & $100 \%$ & 1.55923 & 318353710 & 3502354 \\
\hline 2 & 75.59142 & $53.34 \%$ & 1.27017 & 169794198 & 2853071 \\
\hline 4 & 43.08413 & $30.40 \%$ & 4.14568 & 96776003 & 9312067 \\
\hline 8 & 25.00103 & $17.64 \%$ & 2.58287 & 56157567 & 5801661 \\
\hline 16 & 17.28079 & $12.19 \%$ & 1.61762 & 38816286 & 3633518 \\
\hline 32 & 10.67084 & $7.53 \%$ & 0.51039 & 23968946 & 1146444 \\
\hline
\end{tabular}

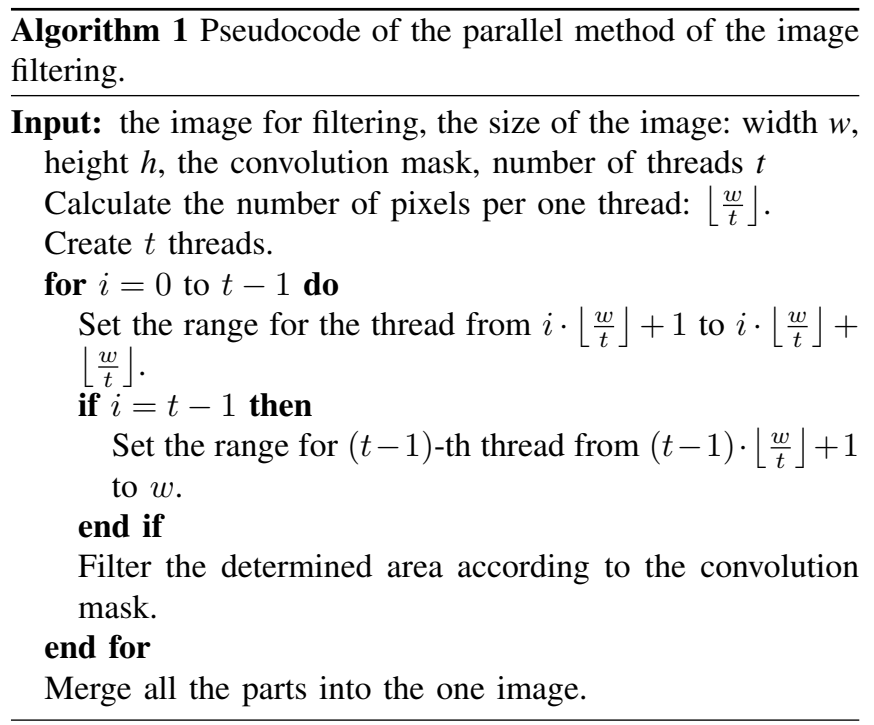

It is possible to observe that even the division of the image into 2 rectangles speeds up significantly the calculations (53\%$57 \%$ of the calculation time for one thread). In the case of the $9 \times 9$ convolution mask, the time was decreased from 141 to 75 seconds. Fig. 5 - 7 show a hyperbolic decline of the computing time. The differences between consecutive cases are getting smaller but still the most beneficial application is while using 32 threads (the maximum number of available CPU threads). The larger the convolution mask is, the more time can be saved thanks to multithreading (7-10 seconds in the case of the $3 \times 3$ mask, 22-42 seconds in the matter of the $5 \times 5$ mask and 66-131 seconds regarding to the $9 \times 9$ mask). As we have proposed multithreading method can be a very useful tool in speeding up the calculations.

\section{CONCLUSIONS AND FINAL REMARKS}

The research has shown that proposed parallelization significantly decreases the time of calculations. The profit is best visible when all CPU threads are used. The larger the picture and the computation mask is, the more important reduction in time spent for calculations is visible. It is worth to mention that the time necessary to filter the image of a size $1200 \times 676$ pixels by using only one thread and the $9 \times 9$ convolution mask exceeds two minutes. It can be concluded that filtering larger image (for instance of a size $6000 \times 6000$ pixels or even higher) involves several minutes, especially in the case of large convolution masks, also bigger than $9 \times 9$. Striving for a significant reduction of the calculation time is essential.

The method can be further developed by examining other parallel algorithms or trying to process many images at the same time. In our future research we will also investigate this methodology in movie processing, since this application can be the most important for HD multimedia systems.

\section{ACKNOWLEDGMENT}

Authors acknowledge contribution to this project of the "Diamond Grant 2016" No. 0080/DIA/2016/45, and from the program "Best of the Best 3.0" both from the Polish Ministry of Science and Higher Education.

\section{REFERENCES}

[1] Z. Marszałek, "Parallel fast sort algorithm for secure multiparty computation," J. UCS, vol. 24, no. 4, pp. 488-514, 2018.

[2] Z. Marszalek, "Parallelization of modified merge sort algorithm," Symmetry, vol. 9, no. 9, p. 176, 2017, DOI: 10.3390/sym9090176.

[3] J. Protasiewicz, "Inventorum: A platform for open innovation," in Systems, Man, and Cybernetics (SMC), 2017 IEEE International Conference on. IEEE, 2017, pp. 10-15. 


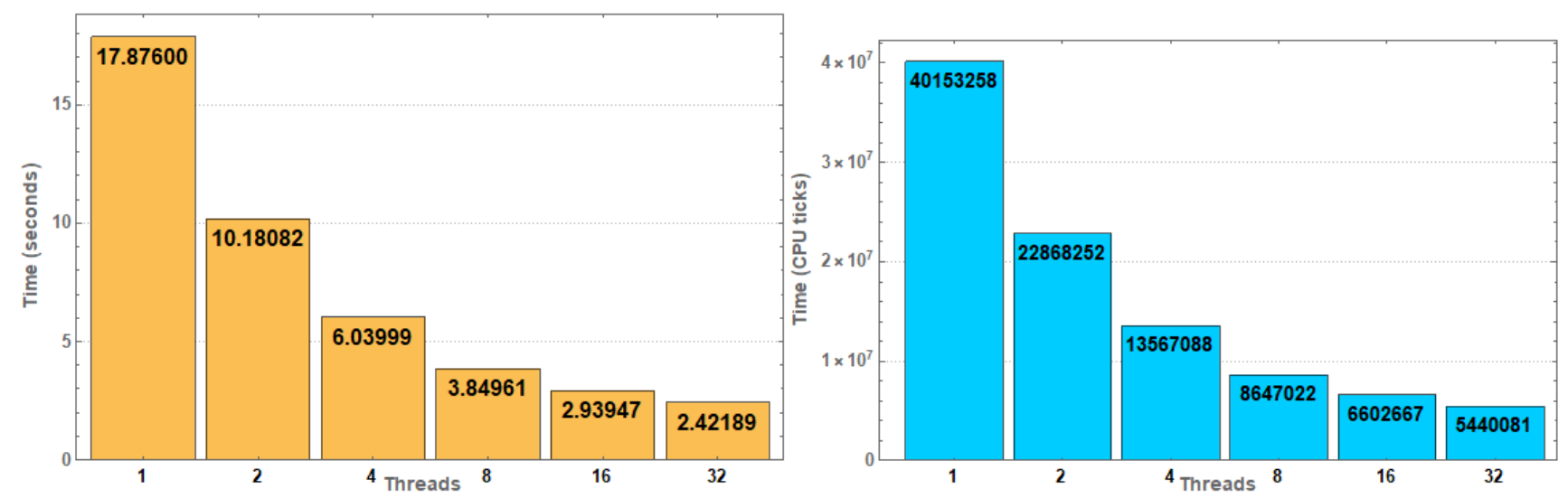

Fig. 5: Results for $3 \times 3$ convolution mask.
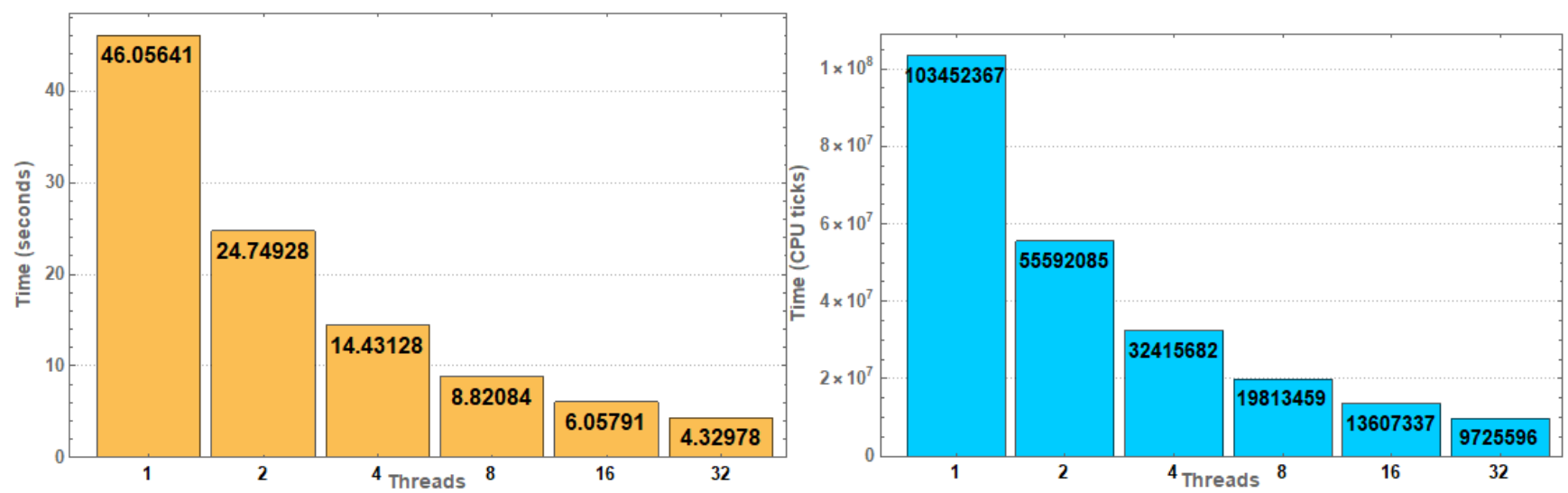

Fig. 6: Results for $5 \times 5$ convolution mask.
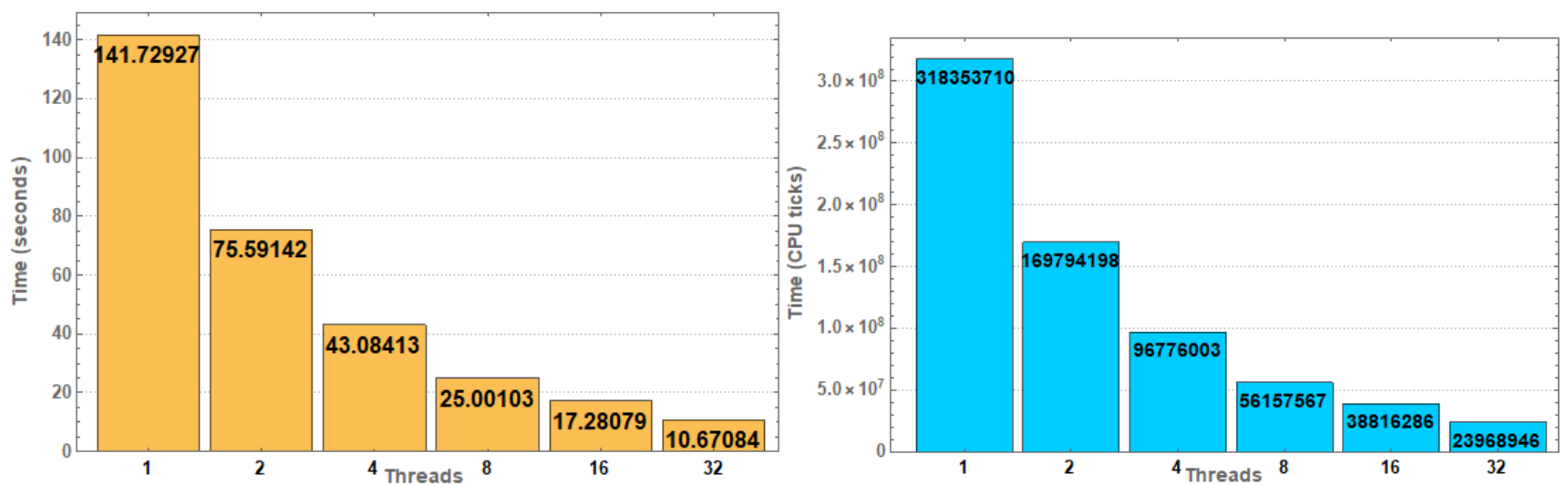

Fig. 7: Results for $9 \times 9$ convolution mask.

[4] D. D. Burdescu, L. Stanescu, M. Brezovan, F. Slabu, and D. Ebanca "Multimedia data for efficient detection of visual objects," in Proceedings of the 11th International Conference on Ubiquitous Information Management and Communication, ser. IMCOM '17. New York, NY, USA: ACM, 2017, pp. 61:1-61:8, DOI: 10.1145/3022227.3022287.

[5] D. D. Burdescu, M. Brezovan, L. Stănescu, C. S. Spahiu, and D. C. Ebâncă, "Graph-based semantic segmentation for 3d digital images," in
201731 st International Conference on Advanced Information Networking and Applications Workshops (WAINA), 2017, pp. 114-119, DOI: 10.1109/WAINA.2017.69.

[6] Y. Jia, C. Rong, C. Wu, and Y. Yang, "Research on the decomposition and fusion method for the infrared and visible images based on the guided image filtering and gaussian filter," in 2017 3rd IEEE International Conference on Computer and Communications (ICCC), 2017, pp. 
1797-1802, DOI: $10.1109 /$ CompComm.2017.8322849.

[7] S. Deniziak and T. Michno, "New content based image retrieval database structure using query by approximate shapes," in Proceedings of the 2017 Federated Conference on Computer Science and Information Systems, FedCSIS 2017, Prague, Czech Republic, September 3-6, 2017. 2017, pp. 613-621, DOI: 10.15439/2017F457.

[8] —, "Query-by-shape interface for content based image retrieval," in 8th International Conference on Human System Interaction, HSI 2015, Warsaw, Poland, June 25-27, 2015, 2015, pp. 108-114, DOI: 10.1109/HSI.2015.7170652.

[9] R. Karumuri and S. A. Kumari, "Weighted guided image filtering for image enhancement," in 2017 2nd International Conference on Communication and Electronics Systems (ICCES), 2017, pp. 545-548, DOI: 10.1109/CESYS.2017.8321137

[10] N. Dhengre, K. P. Upla, H. Patel, and V. M. Chudasama, "Biomedical image fusion based on phase-congruency and guided filter," in 2017 Fourth International Conference on Image Information Processing (ICIIP), 2017, pp. 1-5, DOI: 10.1109/ICIIP.2017.8313792.

[11] C. Chen and M. C. Stamm, "Image filter identification using demosaicing residual features," in 2017 IEEE International Confer- ence on Image Processing (ICIP), 2017, pp. 4103-4107, DOI 10.1109/ICIP.2017.8297054

[12] S. K. Dewangan, "Visual quality restoration enhancement of underwater images using hsv filter analysis," in 2017 International Conference on Trends in Electronics and Informatics (ICEI), 2017, pp. 766-772, DOI 10.1109/ICOEI.2017.8300807.

[13] E. Royer, J. Chazalon, M. Rusiñol, and F. Bouchara, "Benchmarking keypoint filtering approaches for document image matching," in 2017 14th IAPR International Conference on Document Analysis and Recognition (ICDAR), vol. 01, 2017, pp. 343-348, DOI: 10.1109/ICDAR.2017.64.

[14] R. Jain, R. Kasturi, and B. G. Schunck, Machine vision. McGraw-Hill New York, 1995, vol. 5.

[15] R. C. Gonzalez and R. E. Woods, Digital image processing. Upper Saddle River, NJ: Prentice Hall, 2012.

[16] Z. Czech, Wprowadzenie do obliczeń równolegtych. Wydawnictwo Naukowe PWN, 2013.

[17] T. Zieliński, Cyfrowe przetwarzanie sygnałów: od teorii do zastosowań. Wydawnictwa Komunikacji i Łaczności, 2007. 